\title{
Strangeness production in hadronic models and recombination models
}

\author{
Gunnar Gräf ${ }^{1}$, Elvira Santini ${ }^{1}$, Hannah Petersen ${ }^{1,2}$, Jan \\ Steinheimer $^{1}$, Michael Mitrovski ${ }^{3}$, Marcus Bleicher ${ }^{3}$ \\ ${ }^{1}$ Institut für Theoretische Physik, Goethe Universität Frankfurt,Germany \\ ${ }^{2}$ Department of Physics, Duke University, Durham, NC 27708, United States \\ ${ }^{3}$ Frankfurt Institute for Advanced Studies (FIAS), Ruth-Moufang-Str. 1, 60438 \\ Frankfurt, Germany \\ E-mail: bleicher@th.physik.uni-frankfurt.de
}

\begin{abstract}
We present recent results on the production, spectra and elliptic flow of strange particles in dynamic simulations employing hadronic degrees of freedom and from recombination models. The main focus will be on the Ultra-relativistic Molecular Dynamics (UrQMD) Boltzmann approach to relativistic heavy ion collisions and a hybrid approach with intermediate hydrodynamic evolution based on UrQMD (available for download as UrQMD v3.3). Compared to the standard binary collision approach, an enhancement of the strange particle particle yields is found in the hybrid approach due to the assumption of local equilibration. The production origins of the $\phi$-meson in the hybrid approach are studied in further detail. We also present results on the transverse momentum spectra of baryon to meson ratios of strange particles. Due to the approximate energy independent scaling of this ratio as a function of $p_{T}$ we argue, that a maximum in these spectra may not be a unique sign for quark coalescence but can be understood in terms of flow and fragmentation.
\end{abstract}




\section{Introduction}

Heavy ion collisions offer the unique possibility to explore the properties of nuclear matter under extreme conditions in the laboratory. To achieve high temperatures and particle densities, accelerators like the Relativistic Heavy Ion Collider (RHIC) and Large Hadron Collider (LHC) or the planned FAIR facility are used to collide heavy nuclei and to detect the remnants of their interaction. During the collision of these nuclei it is expected that a hot and dense zone of free quarks and gluons, known as the quark gluon plasma (QGP) is created.

Many years ago, strangeness has been proposed as a good tool to investigate this new state of matter [1], because the strange $(s)$ and anti-strange $(\bar{s})$ quarks are not present in the colliding nuclei and have to be produced during the collision. An additional advantage of strange quarks is that they decay by weak interactions, which means that they have a lifetime on the order of $10^{-10}$ seconds which is much longer than the timescale of a heavy ion collision and allows for a direct reconstruction of strange hadrons in the detectors.

On the theoretical side, the production of multi-strange (anti-)baryons and $\phi$ mesons is still a difficult task. Hadronic transport approaches based on binary particle scatterings (i.e. without a QGP transition or additional effects like strong color fields [2] or multi-particle interactions [3, 4]) are usually not able to reproduce the multiplicities of multi-strange hadrons. This observation is related to the long equilibration times and high mass threshold in the hadronic environment compared to a QGP state.

Hydrodynamics has been suggested as an alternative tool to describe the hot and dense stage of heavy ion collisions [5, 6, 7, 8, 9, 10, 11]. Here one assumes local equilibration of the QCD-matter, however, one has to deal with complications concerning the decoupling stage and potential off-equilibrium effects, like viscosities or heat conductivity. In these proceedings we present a novel integrated hybrid approach that dynamically links a non-equilibrium Boltzmann dynamics approach for the initial and final stage of the reaction to an intermediate ideal hydrodynamic stage. This integrated hybrid approach is called UrQMD v3.3 [12] and is based on the known Ultrarelativistic Quantum Molecular Dynamics. For a detailed description of the model and comparison to data the reader is referred to [13, 14, 15, 16].

\section{Hybrid approaches}

Hybrid approaches to unite hydrodynamics and transport equations where proposed 10 years ago [17, 18] and have since then been employed for a wide range of investigations $[19,20,21,22$. The hybrid approach presented here is based on the integration of a hydrodynamic evolution into the UrQMD approach [23]. During the first phase of the evolution the particles are described by UrQMD as a purely hadronic cascade. Once the two colliding nuclei have passed through each other the hydrodynamic evolution starts at the time $t_{\text {start }}=2 R / \sqrt{\gamma^{2}-1}$. At this time the spectators continue to propagate in 

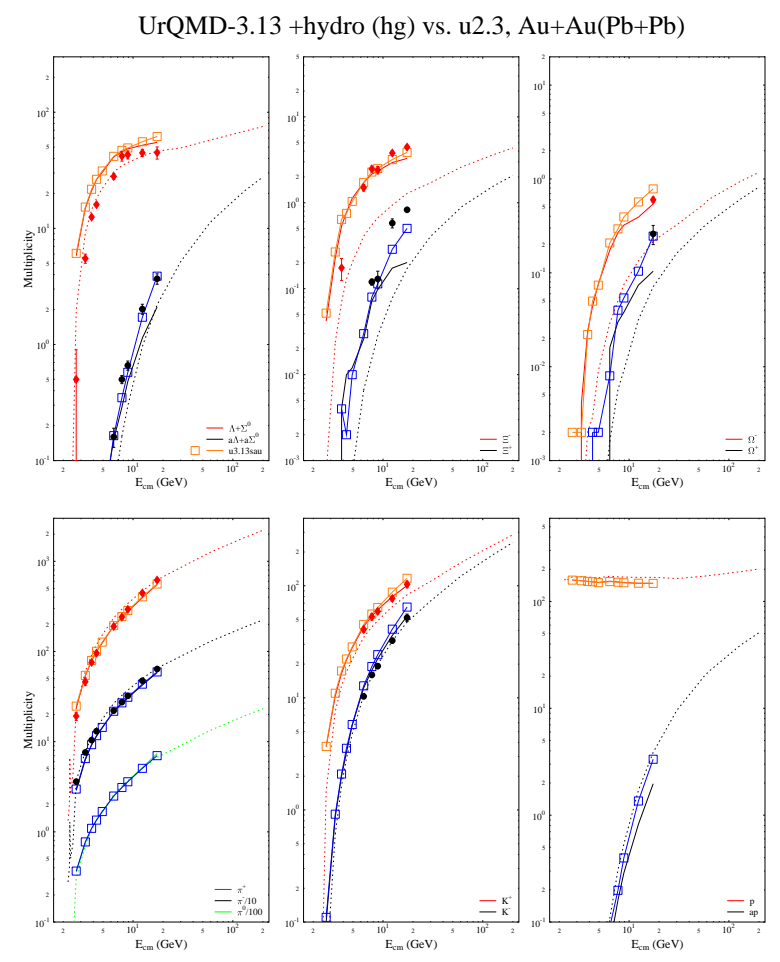

Figure 1. Excitation functions of the $\Lambda, \Sigma, \Omega, \pi, K$ and $p$ multiplicities $(4 \pi)$ in central $(b<3 . f \mathrm{fm}) \mathrm{Au}+\mathrm{Au} / \mathrm{Pb}+\mathrm{Pb}$ collisions. The results for the hybrid model with isochronous freeze-out (full lines), the hybrid model with gradual freeze-out (squares) and pure UrQMD-2.3 (dotted lines) are compared to experimental data from various experiments.

the cascade, while all other particles are mapped to the hydrodynamic grid. By doing so one explicitly assumes a local thermal equilibrium for each cell. The hydrodynamic evolution is performed using the SHASTA [6] algorithm with a hadron gas equation of state. At the end of this phase the hydrodynamic fields are mapped to particle degrees of freedom using the Cooper-Frye equation. Two possible freeze-out criteria procedures where tested. The first is the isochronous freeze-out (IF). In this approach, all hydrodynamic cells are mapped onto particles at the same time, once the energy drops below five times the ground state energy density in all cells. The second approach is called gradual freeze-out (GF). In this approach the freeze-out happens simultaneously in a transverse slice of thickness $0.2 \mathrm{fm}$ once all cells in this slice have an energy density lower than five times the ground state energy. This mimics an iso-eigentime freeze-out that accounts for time dilation effects seen in the freeze-out temperature in cells with a high rapidity. After this the final state interactions and decays of the particles happen within the UrQMD framework.

In Fig. 1 the excitation functions of the $\Lambda, \Sigma, \Omega, \pi, K$ and $p$ yields are shown. 
One observes an enhancement of the multiplicities for all particles with strange content compared to the non-hybrid results. This enhancement leads to an improved description of the experimental data. This is especially true for the gradual freeze-out, while the isochronous freeze-out suffers from relativistic effects at higher beam energies. For further discussion on spectra, the reader is referred to [13.

\section{3. $\phi$-production}

Apart from multi-strange baryons, $\phi$-mesons pose a second problem in many conventional hadronic approaches. This difficulty is not restricted to ultra-relativistic collision energies, but is also present at lower reaction energies, see e.g. 24]. Recently, the present hybrid approach has been used to investigate $\phi \rightarrow \mu^{+} \mu^{-}$production in In + In collisions at $E_{\text {lab }}=158 \mathrm{AGeV}$ [25]. It was found that the hybrid model naturally provides a realistic description of the $\phi$ yields and their transverse mass spectra. In Fig. 2 (left), the hybrid model calculations are compared to pure cascade calculations, in which no assumption of an intermediated locally equilibrated phase is made, and to NA60 data [26]. The two extreme cases of central and peripheral collisions are considered. We observe that differences in the transverse dynamics resulting from the two approaches become stronger especially for central collisions, with NA60 data favouring the hybrid approach to the pure transport calculation. Small deviations between the hybrid approach calculations and the experimental data are found for peripheral collisions where this kind of hybrid models seem to reach their limit of applicability [14]. The transverse dynamics resulting from pure transport calculations is comparable to the experimental data for the peripheral reaction, though the yield remains underestimated. In this respect, however, it should be mentioned that the UrQMD model underestimates $\phi$ meson production at high energies already in $p+p$ collisions. This might partially explain that the main deviations between the experimental data and the transport calculations for peripheral reactions are essentially related to the yield.

Let us now discuss more in detail the hybrid model calculations and, in particular, the emission during the cascade stage. In Fig. 2 (right) a decomposition of the various contributions from the cascade emission is shown. This stage contains pre- and postequilibrium emission. The pre-equilibrium di-muon emission is negligible, as expected from the shortness of this stage. The post-equilibrium emission can be divided in two categories: (i) the emission from $\phi$ particles produced via Cooper-Frye at the transition point and (ii) the emission from $\phi$ particles produced during the cascade.

In the first case, the particles have a momentum distribution that reflects the thermal properties of the transition point, although their di-muon decay occurs later in

a non-thermal environment. In this sense, this copious emission, though not specifically thermal (i.e. not described by thermal rate equations) still carries information about the preceding thermal phase.

The second contribution, on the contrary, can be labeled as a "purely cascade" one. This is the contribution of $\phi$ particles produced in the non-equilibrium environment 

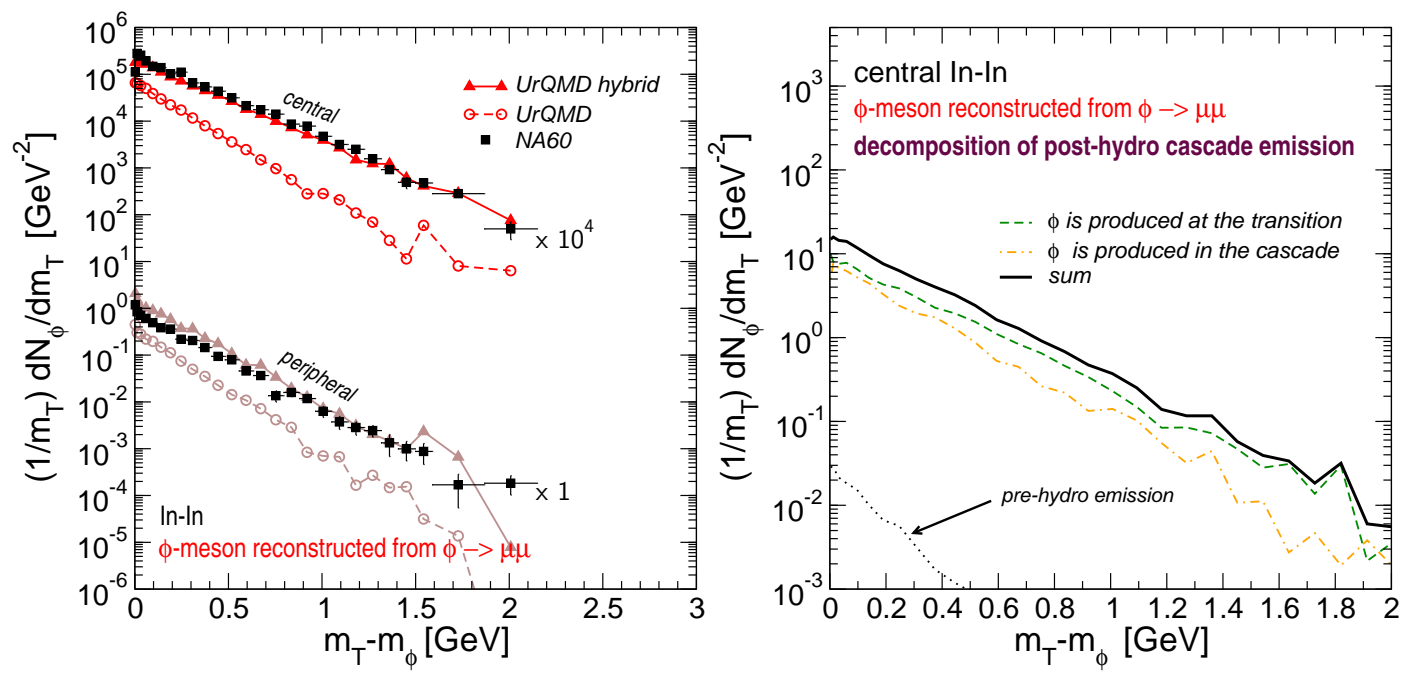

Figure 2. Left: Transverse mass distributions of the $\phi$ meson in central (top) and peripheral (bottom) indium-indium collisions. The hybrid model calculation (full line) is compared to the pure UrQMD transport calculation (dashed line) and to experimental data [26. Bin-widths which coincide with the ones of the experimental data have been used. Right: Decomposition of the post-equilibrium $\phi \rightarrow \mu^{+} \mu^{-}$ production in: (i) emission from $\phi$ mesons which are merged into the cascade at the transition point via the Cooper-Frye equation and emit in the cascade stage of the evolution (dashed line); (ii) emission from $\phi$ mesons which are produced and emit in the cascade stage (dotted-dashed line). The pre-equilibrium emission is denoted by the dotted line. The full line represents the total cascade emission. Due to the smallness of the pre-equilibrium emission the latter practically coincides with the total post-equilibrium emission.

on the way to final decoupling. This second contribution is characterised by steeper transverse mass spectra. the shape of the total spectra is found to be composed by the interplay of both emissions. Emission during the hydrodynamical phase of the evolution has been investigated too and was found to be smaller than the post-cascade emission [25].

The analysis supports the picture of a $\phi$ meson being emitted from an equilibrium source. The mechanism behind the thermalization of the $\phi$ meson cannot be inferred by this analysis and remains an open question. The possible mechanism for the (eventual) thermalization of the $\phi$ in medium are still under debate. In transport calculations the dynamics of the $\phi$ meson is typically characterised by small cross sections and early transport calculations [27, 28] supported the idea of a large $\phi$ mean free path in the hot hadronic fireball (see also [29]). In the UrQMD model, more specifically, the $\phi$ meson couples mainly to $K \bar{K}$ and $\rho \pi$ channels and its elastic and inelastic scattering with mesons and baryons are modelled within the Additive Quark Model. Values for the cross sections for such reactions can be found in Table IX of Ref. [30]. Over the years, however, some mechanisms have been suggested that could be responsible for a stronger $\phi$ coupling to the high density high temperature phase than expected. Alvarez-Ruso and Koch, for example, studied the interactions of the $\phi$ meson with other pseudo-scalar 

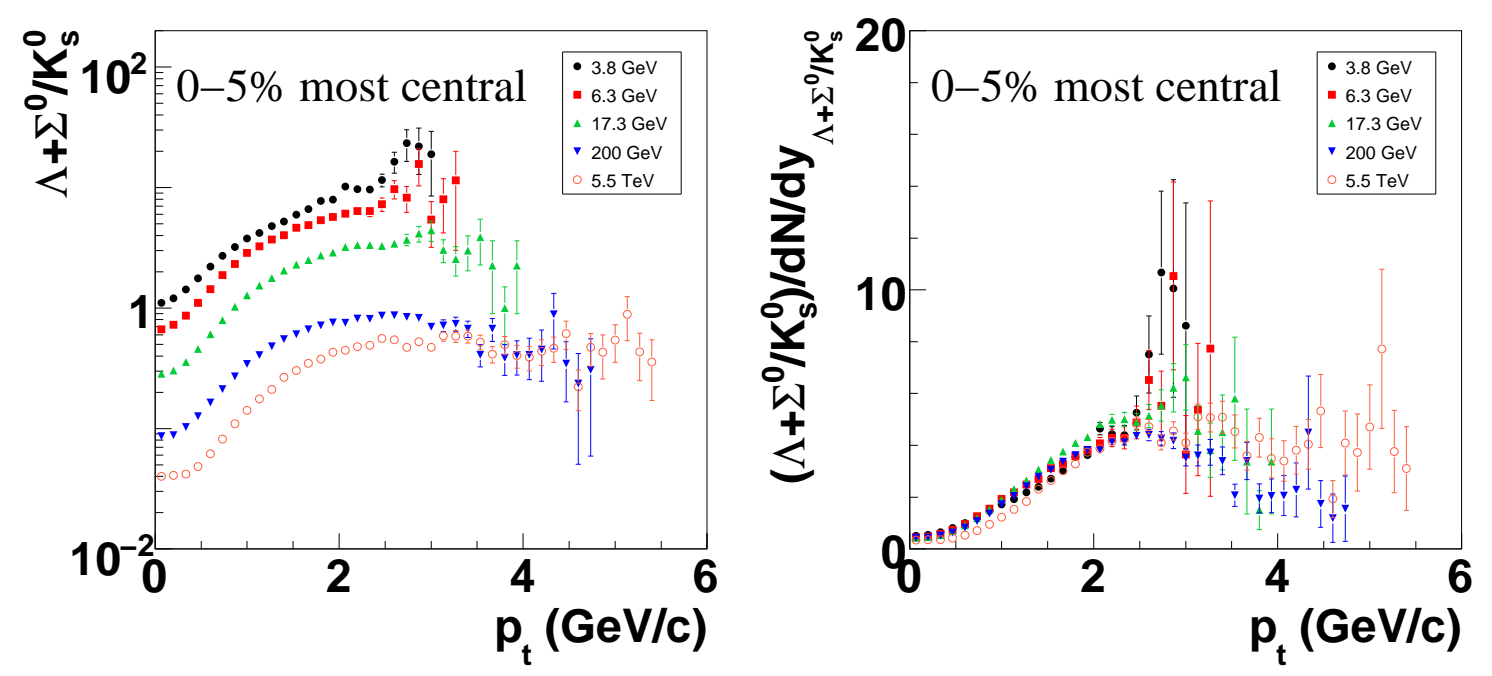

Figure 3. Left: Transverse momentum distribution at midrapidity $|y| \leq 0.5$ of the $\left(\Lambda+\Sigma^{0}\right) / K_{s}^{0}$ ratio from $\sqrt{s}=3.8 \mathrm{GeV}$ (top) to $\sqrt{s}=5.5 \mathrm{TeV}$ (bottom). The values were calculated with pure UrQMD-2.3 for $0-5 \%$ most central $\mathrm{Au}+\mathrm{Au}$ collisions. Right: Transverse momentum distribution at midrapidity of the $\left(\Lambda+\Sigma^{0}\right) / K_{s}^{0}$ ratio scaled with the yield at midrapidity $|y| \leq 0.5$ for center of mass energies from $3.8 \mathrm{GeV}$ to 5.5 $\mathrm{TeV}$.

and vector mesons in a hadronic gas at finite temperature [31. Within the Hidden Local Symmetry Lagrangian they calculate the $\phi$ collision rate and mean free path including an extended family of mechanisms that are allowed by symmetries but are not present in calculations that rely on observed decays. In such a scenario a significantly smaller mean free path (between 2.4 and $1 \mathrm{fm}$ at temperatures above $T=170 \mathrm{MeV}$ ) was found. Recently, the hypothesis of a catalytic $\phi$ meson production in heavy-ion collisions has been suggested [32]. When implemented within a schematic fireball evolution scenario, such an hypothesis can account for the increase of the $\phi$ rapidity distribution width with the collision energy. In a recent calculation of spectral density of the $\phi$ meson in a hot bath of nucleons and pions [33] a significant increase of the width of the $\phi$ meson in medium, which again supports the scenario of a $\phi$ not at all weakly coupled to the medium. Another possibility within the hadronic scenario might be multi-particle interaction that could as well contribute to increase the degree of thermalization and lead to thermalized $\phi$ production [34].

\section{Baryon to meson ratios}

At the Relativistic Heavy Ion Collider (RHIC) at Brookhaven an unexpected enhancement of the baryon to meson ratios in central $\mathrm{Au}+\mathrm{Au}$ reactions at $\sqrt{s}=200 \mathrm{GeV}$ in the transverse momentum region about $2 \mathrm{GeV} / \mathrm{c}$ had been observed. Fries et al. looked in to the baryon to meson ratios [35] using a combined recombination and fragmentation picture. Comparing their results with statistical models and data they 
suggest that the maximum in the $p_{\perp}$ dependence of $\mathrm{p} / \pi^{0}$ and $\Lambda / K^{0}$ ratios is a strong sign for a transition of a recombination dominated regime to a fragmentation dominated regime at higher $p_{\perp}$.

Let us investigate this effect in Fig. 3(left). Here, the results of pure UrQMD calculations without a hydrodynamic phase are shown. One clearly observes a maximum of the baryon to meson ratio at about $2 \mathrm{GeV} / \mathrm{c}$. However, it seems that the magnitude of the enhancement is less pronounced than in the data. To test, if the enhancement is unique to the high $p_{\perp}$ tail or if it results from an overall normalisation, we normalise the $p_{\perp}$ spectra to their integral.

The normalised $\left(\Lambda+\Sigma^{0}\right) / K_{s}^{0}$ ratios are shown in Fig. 3 (right). The surprising result is a collapse of all $\left(\Lambda+\Sigma^{0}\right) / K_{s}^{0}$ ratios at various center of mass energies from $3.8 \mathrm{GeV}$ (lower AGS) to $5.5 \mathrm{TeV}$ (LHC) to a single line. I.e. the apparent maximum of the baryon to meson ratio at $p_{\perp} \approx 2 \mathrm{GeV}$ is present at all investigated energies. We thus conclude, that the maximum in the baryon to meson ratios, may not be a unique sign for quark coalescence, but rather a natural consequence of a flow plus fragmentation picture.

In summary, we have discussed recent developments in the area of hadron transport simulations with emphasis on strangeness production. The most important improvements over the last decade include the wide range employment of hybrid approaches to simulate the hot and dense reaction stage by ideal (and also dissipative) hydrodynamics coupled to fluctuating non-equilibrium initial conditions and followed by a decoupling stage modelled via hadronic cascades. These combined approaches have proven their power over a wide range of applications ranging from strange particle production, over (elliptic) flow analysis to leptonic and photonic probes. At the end of these proceedings we have also shortly presented a critical view on the baryon to meson ratios and their potential as a signal for parton recombination.

\section{Acknowledgements}

This work was supported by the Helmholtz International Center for FAIR within the framework of the LOEWE program launched by the State of Hesse, GSI, BMBF and DESY. The hydrodynamic model is provided by Dirk Rischke. The calculations have been computed at the Center for Scientific Computing at Goethe University.

[1] P. Koch, B. Muller and J. Rafelski, Phys. Rept. 142, 167 (1986).

[2] M. Bleicher, W. Greiner, H. Stoecker and N. Xu, Phys. Rev. C 62, 061901 (2000) arXiv:hep-ph/0007215.

[3] C. Greiner and S. Leupold, J. Phys. G 27, L95 (2001) arXiv:nucl-th/0009036.

[4] W. Cassing, Nucl. Phys. A 700, 618 (2002) arXiv:nucl-th/0105069].

[5] L. P. Csernai, I. Lovas, J. A. Maruhn, A. Rosenhauer, J. Zimanyi and W. Greiner, Phys. Rev. C 26, 149 (1982). 
[6] D. H. Rischke, S. Bernard and J. A. Maruhn, Nucl. Phys. A 595, 346 (1995) arXiv:nucl-th/9504018.

[7] D. H. Rischke, Y. Pursun and J. A. Maruhn, Nucl. Phys. A 595, 383 (1995) [Erratum-ibid. A 596, 717 (1996)] [arXiv:nucl-th/9504021].

[8] D. H. Rischke, Nucl. Phys. A 610, 88C (1996) arXiv:nucl-th/9608024].

[9] T. Hirano, Phys. Rev. C 65, 011901 (2002) arXiv:nucl-th/0108004.

[10] P. F. Kolb and U. W. Heinz, arXiv:nucl-th/0305084.

[11] P. Huovinen and D. Molnar, Phys. Rev. C 79, 014906 (2009) [arXiv:0808.0953 [nucl-th]].

[12] Website of the UrQMD collaboration: http://urqmd.org

[13] H. Petersen, J. Steinheimer, G. Burau, M. Bleicher and H. Stocker, Phys. Rev. C 78, 044901 (2008) arXiv:0806.1695 [nucl-th]].

[14] H. Petersen, M. Mitrovski, T. Schuster and M. Bleicher, Phys. Rev. C 80, 054910 (2009) arXiv:0903.0396 [hep-ph]].

[15] H. Petersen and M. Bleicher, Phys. Rev. C 79, 054904 (2009) arXiv:0901.3821 [nucl-th]].

[16] H. Petersen, J. Steinheimer, M. Bleicher and H. Stocker, J. Phys. G 36, 055104 (2009) arXiv:0902.4866 [nucl-th]].

[17] S. A. Bass, A. Dumitru, M. Bleicher, L. Bravina, E. Zabrodin, H. Stoecker and W. Greiner, Phys. Rev. C 60, 021902 (1999) arXiv:nucl-th/9902062.

[18] A. Dumitru, S. A. Bass, M. Bleicher, H. Stoecker and W. Greiner, Phys. Lett. B 460, 411 (1999) arXiv:nucl-th/9901046.

[19] D. Teaney, J. Lauret and E. V. Shuryak, arXiv:nucl-th/0110037.

[20] O. . J. Socolowski, F. Grassi, Y. Hama and T. Kodama, Phys. Rev. Lett. 93, 182301 (2004) arXiv:hep-ph/0405181.

[21] C. Nonaka and S. A. Bass, Nucl. Phys. A 774, 873 (2006) arXiv:nucl-th/0510038.

[22] T. Hirano and M. Gyulassy, Nucl. Phys. A 769, 71 (2006) arXiv:nucl-th/0506049.

[23] H. Petersen, M. Bleicher, S. A. Bass and H. Stocker, arXiv:0805.0567 [hep-ph].

[24] H. W. Barz and B. Kampfer, Nucl. Phys. A 683, 594 (2001) arXiv:nucl-th/0005063.

[25] E. Santini, H. Petersen and M. Bleicher, arXiv:0909.4657 [nucl-th].

[26] R. Arnaldi et al. [NA60 Collaboration], Eur. Phys. J. C 64, 1 (2009) arXiv:0906.1102 [hep-ex]].

[27] C. M. Ko and D. Seibert, Phys. Rev. C 49, 2198 (1994) arXiv:nucl-th/9312010.

[28] K. Haglin, Nucl. Phys. A 584, 719 (1995) arXiv:nucl-th/9410028.

[29] H. van Hecke, H. Sorge and N. Xu, Phys. Rev. Lett. 81, 5764 (1998) arXiv:nucl-th/9804035.

[30] M. Bleicher et al., J. Phys. G 25, 1859 (1999) arXiv:hep-ph/9909407.

[31] L. Alvarez-Ruso and V. Koch, Phys. Rev. C 65, 054901 (2002) arXiv:nucl-th/0201011.

[32] E. E. Kolomeitsev and B. Tomasik, J. Phys. G 36, 095104 (2009) arXiv:0903.4322 [nucl-th]].

[33] G. Vujanovic, J. Ruppert and C. Gale, Phys. Rev. C 80, 044907 (2009) arXiv:0907.5385 [nucl-th]].

[34] P. Braun-Munzinger, J. Stachel and C. Wetterich, Phys. Lett. B 596, 61 (2004) arXiv:nucl-th/0311005.

[35] R. J. Fries, B. Muller, C. Nonaka and S. A. Bass, Phys. Rev. C 68, 044902 (2003) arXiv:nucl-th/0306027. 